\title{
New evidence in cardiovascular medicine, general practice and public health
}

\author{
Andrea Alberto Conti · Beatrice Dilaghi · \\ Pietro Amedeo Modesti · Carlo Nozzoli
}

Published online: 9 June 2009

(C) SIMI 2009

\section{Normal systolic blood pressure and very low low-density lipoprotein cholesterol are linked to the slowest progression of coronary atherosclerosis}

Scientific evidence is available with reference to the major role of both blood pressure and low-density lipoprotein cholesterol (LDL-C) in the promotion of coronary artery disease, yet the effect of the concomitant correct control of blood pressure and LDL-C in human beings has not been clarified. A group of US researchers has therefore studied the progression of coronary atheroma in subjects with low LDL$\mathrm{C}$ and variable values of systolic blood pressure. The changes in the burden of atheroma were investigated by means of intravascular ultrasound in 3,437 patients affected by coronary artery disease subdivided into four groups: individuals with LDL-C $\leq 70 \mathrm{mg} / \mathrm{dl}$ and systolic blood pressure $\leq 120 \mathrm{mmHg}$, individuals with LDL-C $\leq 70 \mathrm{mg} / \mathrm{dl}$ and systolic blood pressure $>120 \mathrm{mmHg}$, individuals with LDL$\mathrm{C}>70 \mathrm{mg} / \mathrm{dl}$ and systolic blood pressure $\leq 120 \mathrm{mmHg}$, individuals with LDL-C $>70 \mathrm{mg} / \mathrm{dl}$ and systolic blood pressure $>120 \mathrm{mmHg}$. People with very low LDL-C $(\leq 70 \mathrm{mg} / \mathrm{dl})$ and normal systolic blood pressure $(\leq 120 \mathrm{mmHg})$ showed a more frequent plaque regression

A. A. Conti $(\bowtie) \cdot$ P. A. Modesti

Department of Critical Care Medicine and Surgery,

University of Florence and Don Carlo Gnocchi Foundation, IRCCS Florence, Viale Morgagni 85, 50134 Florence, Italy e-mail: aa.conti@dac.unifi.it

\section{B. Dilaghi}

Department of Emergency Medicine,

Azienda Ospedaliero-Universitaria Careggi, Florence, Italy

C. Nozzoli

General Medicine Unit, Azienda Ospedaliero-Universitaria

Careggi, Florence, Italy
$(P=0.01)$, less progression in percentage atheroma volume $(P<0.001)$ and total atheroma volume $(P<0.001)$, and a less frequent plaque progression $(P<0.001)$. In subjects with systolic blood pressure $>120 \mathrm{mmHg}$, very low LDL-C was linked to less progression of percentage atheroma volume $(+0.30 \%, 95 \%$ confidence interval between -0.17 and $0.77 \%$ versus $+0.61 \%, 95 \%$ confidence interval between 0.17 and $1.05 \%, P=0.01)$ and total atheroma volume $\left(-3.9 \mathrm{~mm}^{3}, 95 \%\right.$ confidence interval between -7.24 and $-0.63 \mathrm{~mm}^{3}$ versus $-1.2 \mathrm{~mm}^{3}, 95 \%$ confidence interval between -4.31 and $1.92 \mathrm{~mm}^{3}, P=0.001$ ). In coronary patients with LDL-C $>70 \mathrm{mg} / \mathrm{dl}$, normal systolic blood pressure was not associated with less progression of percentage atheroma volume or total atheroma volume.

The authors conclude that normal systolic blood pressure and very low low-density lipoprotein cholesterol are linked to the slowest progression of coronary atherosclerosis.

\section{Reference}

Chhatriwalla AK et al (2009) Low levels of low-density lipoprotein cholesterol and blood pressure and progression of coronary atherosclerosis. J Am Coll Cardiol 53:1110 1115

\section{Analysis of the evidence on which ACC/AHA clinical practice guidelines are elaborated}

Recently a group of US researchers have discussed the distribution of the recommendations included in the joint cardiovascular guidelines of the American College of Cardiology (ACC) and of the American Heart Association (AHA). To illustrate the evolution of such recommendations 
the staff in the ACC Science and Quality Division summarized information from the ACC/AHA practice guidelines published from 1984 to 2008, and more than 50 guidelines, with more than 7,000 recommendations were evaluated. The researchers established the total number of recommendations and their subdivision into classes of recommendations (I, II, and III) and levels of evidence (A, B, and C). The total number of recommendations in guidelines with at least one revision by September 2008 passed from 1330 to 1973 $(+48 \%)$ from the first to the current version, and the most remarkable increase in number was detected in class II recommendations. In the 16 current guidelines including the indication of the levels of evidence, 314 recommendations out of 2,711 were classified as level of evidence $A$, and 1,246 were classified as level of evidence $\mathrm{C}$. The recommendations with level of evidence A were predominantly located in class I, even if only 245 out of 1,305 class I recommendations had a level of evidence A. The authors of this study conclude that the recommendations included in currently published ACC/ AHA clinical practice guidelines are often based on lower levels of evidence and that the percentage of recommendations based on evidence which is not conclusive is on the increase. This analysis of the evidence on which ACC/AHA clinical practice guidelines are elaborated therefore indicates, in the opinion of the authors, that the procedure of guidelines writing may and should improve.

\section{Reference}

Tricoci P et al (2009) Scientific evidence underlying the ACC/AHA clinical practice guidelines. JAMA 301:831841

\section{Changes through time in the adherence of European citizens to cardiovascular prevention guidelines}

The recent publication of the third EUROASPIRE survey makes available the possibility of a structured comparison with the previous cross-sectional studies EUROASPIRE I and II, which indicated the limits of cardiovascular prevention in Europe. This last EUROASPIRE survey was conducted in more than 20 European countries not only to compare the control of cardiovascular risk factors across different realities, but also to evaluate the degree to which the Joint European Societies' recommendations on cardiovascular disease prevention are really followed in the real world. EUROASPIRE surveys included consecutive male and female patients with less than 71 years of age, who had undergone percutaneous coronary interventions or surgical cardiac revascularization, or a hospital recovery because of myocardial infarction. More than 3,000 people were interviewed 6 months after the clinical event in EUROASPIRE I, almost 3,000 in the second survey and 2,392 in the third one.

Comparing the sequential surveys, the proportion of (self-reported) diabetes mellitus increased from 17.4 to $20.1 \%$ and to $28.0 \%(P=0.004)$. The frequency of obesity passed from $25.0 \%$ in the first survey to $32.6 \%$ in the second and to $38.0 \%$ in the third $(P=0.0006)$. The frequency of patients with hypertension was relatively steady (58.1\% in EUROASPIRE I, $58.3 \%$ in II, and $60.9 \%$ in III; $P=0.49)$ and the same applied to the percentage of smokers $(20.3 \%$ in EUROASPIRE I, $21.2 \%$ in II, and $18.2 \%$ in III; $P=0.64$ ), even if the frequency of under 50 smoking females increased. The percentage of survived patients with total cholesterol $\geq 200 \mathrm{mg} / \mathrm{dl}$ continuously decreased from $94.5 \%$ in EUROASPIRE I to $76.7 \%$ in II, and $46.2 \%$ in III $(P<0.0001)$.

Taking into account that EUROASPIRE I was completed in 1996, EUROASPIRE II in 2001 and EUROASPIRE III in 2007, their results indicate that, despite partial improvements in smoking rates and in lipid profile control have been recorded in the last 15 years, in the near future much has still to be done in the field of secondary cardiovascular prevention.

\section{Reference}

Kotseva K et al (2009) Cardiovascular prevention guidelines in daily practice: a comparison of EUROASPIRE I, II, and III surveys in eight European countries. Lancet 373:929-940

\section{The normalization of glycemia in hyperglycemic myocardial infarction patients is linked to the improvement of clinical outcomes}

The authors of this study investigated 7,820 patients with acute myocardial infarction and high glucose values on admission $(140 \mathrm{mg} / \mathrm{dl})$ hospitalized between 2000 and 2005 in 40 hospitals of the United States. The enrolled subjects were subdivided on the base of their mean glucose values after admission and were divided into two groups, insulin receivers and insulin non-receivers. To study whether decreased glucose values after admission were independently linked to improved survival, multivariable logistic regression models were elaborated, and propensitymatching methods were adopted to compare the mortality within the hospital between the group of insulin therapy receivers and the one of non-receivers. Following multivariable correction, decreased mean glucose values after admission resulted to be connected with improved survival 
(the odds ratio for mean postadmission glucose levels of 110 to $<140 \mathrm{mg} / \mathrm{dl}$ was 2.1 ; it was 5.3 for glucose values of 140 to $<170$, 6.9 for glucose levels of 170 to $<200$, and 13 for $200 \mathrm{mg} / \mathrm{dl}$ of glycemia, if compared to glucose values $<110 \mathrm{mg} / \mathrm{dl}$ ). In the propensity-matched evaluation the rates of mortality resulted to be comparable with regard to the patients undergoing insulin and non-insulin through the whole array of mean glycemia after admission (range, $P=0.15$ to $P=0.91)$. The researchers conclude that normalization of glycemia in hyperglycemic acute myocardial infarction patients is linked to the improvement of clinical outcomes whether or not they undergo therapy with insulin.

\section{Reference}

Kosiborod M et al (2009) Glucose normalization and outcomes in patients with acute myocardial infarction. Arch Intern Med 169:438-446

\section{The risk of death because of respiratory causes augments in association with an increase in ozone concentration}

The authors of this paper extracted data from the study cohort of the American Cancer Society Cancer Prevention Study II to correlate them with information regarding air pollution coming from 96 metropolitan areas in the United States of America, so as to investigate the impact of the long-term ozone exposure on the mortality connected to air pollution. The data from 448,850 individuals were evaluated, including 118,777 deaths, and information on the maximum daily concentration of ozone was retrieved from 1 April to 30 September for the years 1977-2000. Information regarding the concentration of fine particulate matter was also collected for the years 1999 and 2000. In the models "single-pollutant", higher concentrations of ozone or fine particulate matter PM 2.5 resulted to be associated in a significant way with an increased mortality risk of death because of cardiopulmonary causes. In the models "two-pollutant", ozone resulted associated with the mortality risk because of respiratory causes and fine particulate matter PM 2.5 was associated with the mortality risk because of cardiovascular causes. The relative risk of mortality, because of respiratory causes, linked to an increase in the concentration of ozone of $10 \mathrm{ppb}$ resulted 1.040 (with a 95\% confidence interval included between 1.010 and 1.067). In this analysis a significant increase in the mortality risk because of respiratory causes in connection with increased concentration of ozone was identified, though not an impact of ozone concentration on the mortality risk due to cardiovascular causes was found.

\section{Reference}

Jerrett M et al (2009) Long-term ozone exposure and mortality. N Engl J Med 360:1085-1095

The exposure to secondhand smoke may be associated with an increased risk of cognitive impairment

Aim of this cross-sectional analysis of a population-based study was to investigate the association between salivary cotinine concentration, that is a biomarker of exposure to secondhand smoke, and cognitive decline. Therefore, more than 4,800 non-smoking individuals of at least 50 years of age were studied; they furnished samples of saliva for cotinine assay and a careful smoking history, and in them cognitive impairment was identified as the lowest $10 \%$ of scores of a neuropsychological tests battery. The authors subdivided the recruited subjects, who were not smokers, into four groups, comparable with regard to their size, according to cotinine levels. The odds ratios for cognitive impairment in the second $(0.2-0.3 \mathrm{ng} / \mathrm{ml})$, third $(0.4-$ $0.7 \mathrm{ng} / \mathrm{ml})$, and fourth fourths $(0.8-13.5 \mathrm{ng} / \mathrm{ml})$ of cotinine concentration were, respectively, 1.08 (from 0.78 to 1.48 ), 1.13 (from 0.81 to 1.56 ), and 1.44 (from 1.07 to $1.94 ; P$ for trend 0.02), after correction for an ample array of known risk factors for cognitive decline, as compared to the first fourth of cotinine concentration $(0.0-0.1 \mathrm{ng} / \mathrm{ml})$. Comparable patterns of associations were detected for former and never smokers.

The authors conclude that the exposure to secondhand smoke may be associated with an increased risk of cognitive decline.

\section{Reference}

Llewellyn DJ et al (2009) Exposure to secondhand smoke and cognitive impairment in non-smokers: national cross sectional study with cotinine measurement. BMJ 338: b462

Conflict of interest statement The authors declare that they have no conflict of interest related to the publication of this manuscript. 